\title{
Acquisition of behavioral control by static visual features of an imprinting object: Species generality
}

\author{
LEONARD A. EISERER \\ Franklin and Marshall College, Lancaster, Pennsylvania 17604 \\ and \\ RANDY L. SWOPE \\ Millersville State College, Millersville, Pennsylvania 17551
}

\begin{abstract}
Previous work had indicated that after prolonged exposure to a moving object, ducklings will direct filial responses toward that object even if it remains stationary during its presentation. The present experiment demonstrated the wide species generality of this effect by obtaining similar results in newly hatched chickens, pheasant, turkeys, and quail, as well as ducks.
\end{abstract}

When an arbitrarily selected object is initially presented in motion to a newly hatched duckling, that object rapidly elicits filial behavior (Hoffman, Stratton, Newby, \& Barrett, 1970). Although the object may not initially elicit filial responses if it remains stationary (Hoffman et al., 1970), it will do so after the duckling has received prolonged exposure to the object in motion (Eiserer, 1977). Importantly, the acquisition of behavioral control by the stationary imprinting object can be demonstrated even under conditions in which prolonged exposure to the stationary object itself is not sufficient, that is, when repeated exposure to the object in motion is essential (Hoffman, Eiserer, \& Singer, 1972).

Since presentation of a stationary imprinting object includes all of the static visual features of the object (its particular color, size, and shape), the above-cited research indicates that such features-at least with the kinds of biologically unnatural objects that are used in many imprinting experiments-are essentially neutral in their initial effects upon filial responses but acquire behavioral control as a young bird is exposed to the object in motion. As noted elsewhere (Eiserer, 1978b, 1980), the acquisition of behavioral control by initially neutral features of an imprinting object seems to represent the very essence of infantile attachments to specific surrogate objects. Moreover, the acquisition phenomenon is of great theoretical interest as well, particularly in connection with classical conditioning vs. perceptual learning theories of imprinting (Eiserer, 1978b, 1980).

Despite such empirical and theoretical importance,

This research was supported in part by a grant from the Mellon Foundation. Reprint requests should be sent to the first author at Whitely Psychology Laboratories, Franklin and Marshall College, Lancaster, Pennsylvania 17604. however, apparently the only precocial bird in which the effect has been specifically studied is the duck (Eiserer, 1977, 1980; Eiserer \& Hoffman, 1973, 1974; Eiserer, Hoffman, \& Klein, 1975; Gaioni, Hoffman, DePaulo, \& Stratton, 1978; Hoffman et al., 1972). Klopfer (1965) reported a similar effect in chicks; subjects exposed to a moving object subsequently preferred that object even when it was stationary. However, the control group in that study showed some indication of preference for the stimulus after only $20 \mathrm{~min}$ of exposure to the stationary stimulus, so it is not clear whether or not the visual features of the object (a mallard decoy) were initially neutral.

The present research sought to determine whether the development of filial attachment to initially neutral features of an imprinting object was a phenomenon that had generality across different species of precocial bird. Should the effect prove to be limited to a restricted set of circumstances-idiosyncratic just to imprinting in ducklings, for example-then the relevant empirical and theoretical considerations would seem to lose much of their import.

\section{METHOD}

\section{Subjects}

The subjects included 8 Khaki Campbell ducklings (Anas platyrhynchos), 8 Ringneck pheasant (Phasianas colchicac), 12 White Leghorn chicks (Gallus gallus), 12 domestic turkeys (Meleagris gallopavo), and 10 Japanese quail (Coturnix coturnix). Because of unexpected mortality in the quail, two separate hatches were required to obtain the 10 final subjects.

\footnotetext{
Apparatus

The experimental apparatus consisted of a plywood box $(120$ $\times 66 \times 78 \mathrm{~cm}$ ) that was divided by a fine-mesh stainless steel screen into two compartments, one for the subject $(84 \times 66 \times 78 \mathrm{~cm})$ and the other for the imprinting object $(36 \times 66 \times 78 \mathrm{~cm})$. To permit assessment of the birds' locomotor behavior during testing,
} 
the carpeted subject compartment was itself divided into two unequal portions by a strip of adhesive tape running parallel to, and at a distance $17 \mathrm{~cm}$ from, the fine-mesh screen of the stimulus compartment. Hence, the resulting small and large areas of the subject compartment were $17 \times 66 \mathrm{~cm}$ and $67 \times 66 \mathrm{~cm}$, respectively, with the smaller area (the "approach area") nearest the stimulus compartment.

A second section of fine-mesh screen (i.e., in addition to the screen that separated the subject and stimulus compartments) was built into one of the side walls of the subject compartment. This screen $(36 \mathrm{~cm}$ high $\times 80 \mathrm{~cm}$ long) permitted the experimenter to observe the exact location of a bird while it was in the imprinting apparatus.

Lighting in the subject compartment was provided by two continuously illuminated incandescent lamps $(75-\mathrm{W})$ mounted along the top of the compartment. These lamps were positioned so that unless the imprinting stimulus lights (described below) were also illuminated, the light that reflected from the fine-mesh screen prevented the subject from seeing into the darkened stimulus compartment. The same principle operated to prevent subjects from seeing the human observer.

The imprinting stimulus used with the ducks and pheasant consisted of an amber-colored rotating lamp (of the sort used on the tops of many emergency vehicles), approximately $10 \mathrm{~cm}$ high and $10 \mathrm{~cm}$ in diameter, which was placed in the center of the stimulus compartment. Presentations of the moving stimulus were produced by illuminating the lamp's bulb and rotating its lens system. Presentations of the stationary stimulus were produced by illuminating the lamp's bulb while withholding power from the motor that rotated the lens system. During complete stimulus withdrawal, the bulb was not illuminated and the lens system was not rotated.

Pilot work involving exposure of a few quail to the rotating lamp revealed that the moving stimulus elicited very little approach behavior. In an effort to employ a more adequate releaser of filial behavior for this species, the rotating lamp was replaced by a different imprinting object that consisted of three circular lights (one red, one green, and the third, blue) mounted vertically on a wood panel which moved horizontally along the length of the stimulus compartment. These lights were each $1.2 \mathrm{~cm}$ in diameter and were spaced some $2.5 \mathrm{~cm}$ apart from each other. Presentations of the moving stimulus were produced by illuminating the three colored lights and moving the wood panel back and forth across the stimulus compartment at approximately $5.3 \mathrm{~cm} / \mathrm{sec}$. Presentations of the stationary stimulus were produced by illuminating the three colored lights while withholding power from the motor that moved the wood panel. Complete stimulus withdrawal was accomplished by extinguishing the colored lights and, if necessary, stopping the movement of the wood panel.

The moving light panel, with its different pattern of visual motion and its contrast of colors, proved to be somewhat more effective as an imprinting object for the quail than the rotating lamp had been. On the possibility that the same would hold true for other species, the moving light panel was also employed as the imprinting object for the chickens and turkeys, which were run after work with the quail was completed.

\section{Procedure}

Upon hatching, all of the subjects were maintained in visual isolation from conspecifics. Except for periods spent in the experimental apparatus, each subject was kept in an individual brown rectangular box $(30.5 \times 50.8 \times 30.5 \mathrm{~cm})$ that was partially filled with bedding material. Under these circumstances, the subjects could hear conspecifics, but their visual environment was restricted to that provided by the inside of their individual housing units. While in those units, the birds had continuous access to food and water.

The experimental procedure was the same for all five species. At $10-16 \mathrm{~h}$ posthatch, each subject was assigned randomly to either the moving-stimulus group (M), for which the imprinting object was in continuous motion throughout each exposure session, or the stationary stimulus group (S), for which the imprinting object remained stationary throughout each exposure session. Group sizes for each species were as follows: ducks, $4 \mathrm{M}$ and $4 \mathrm{~S}$; pheasant, $4 \mathrm{M}$ and $4 \mathrm{~S}$; chickens, $6 \mathrm{M}$ and $6 \mathrm{~S}$; turkeys, $6 \mathrm{M}$ and $6 \mathrm{~S}$; and quail, $6 \mathrm{M}$ and $4 \mathrm{~S}$. Although these sample sizes are small relative to those typically employed in the imprinting literature, previous closely related research (Eiserer, 1977, 1980; Eiserer \& Hoffman, 1974; Eiserer et al., 1975; Gaioni et al., 1978; Hoffman et al., 1972) has indicated that the phenomenon of concern is robust enough to bear investigation with a small-n methodology.

Subjects in both groups received a total of nine 20 -min sessions at the rate of 2 sessions/day, beginning 10-16 h posthatch; the two daily sessions were approximately $6 \mathrm{~h}$ apart. Immediately following each session, as well as immediately before the first session, each subject received an approach test. The procedure was identical for both groups: At the start of a given test, the imprinting stimulus remained withdrawn for $60 \mathrm{sec}$, after which the stationary stimulus was presented for $60 \mathrm{sec}$, then withdrawn for $60 \mathrm{sec}$, and finally presented again for $60 \mathrm{sec}$. In order to ensure that the subject had to make an active response to accumulate time in the approach area, the experimenter placed the bird near the center of the subject compartment at the beginning of each of the above four 60 -sec intervals.

An experimenter monitored the position of the subject throughout the tests as well as during a random sample of exposure sessions. Approach was defined as the number of seconds that the subject spent within the area nearest the stimulus compartment, beginning as soon as any portion of the bird's foot touched the strip of tape that bordered the area.

\section{RESULTS}

Except for the quail, the $\mathbf{M}$ subjects of each species generally showed strong and persistent approach responses to the moving stimulus within the first one or two exposure sessions. The quail, however, were much less consistent in their responses to the moving stimulus. During any given session, these birds would run to the moving stimulus and clearly attend to it; then, after a few seconds or a few minutes, they would inevitably abandon the stimulus and dart to and fro about the subject compartment, only to eventually reapproach the imprinting stimulus for a limited time again. The behavioral control exerted by the moving stimulus actually appeared to weaken slightly, rather than strengthen, in the later exposure sessions.

As indicated in Figure 1, the $\mathrm{M}$ duck, pheasant, chicken, and turkey subjects developed strong approach tendencies toward the stationary stimulus as a function of exposure to the moving stimulus. In contrast, the S subjects for these species showed only weak and unstable approach. The $M$ quail did not approach the stationary stimulus as much as the $\mathbf{M}$ subjects of the other species; nevertheless, the $M$ quail did approach the stationary stimulus more than did the $S$ quail during most of the tests.

A separate analysis of variance (two-factor mixed design: repeated measures on one factor; Bruning \& Kintz, 1977, pp. 55-61) was conducted for each 


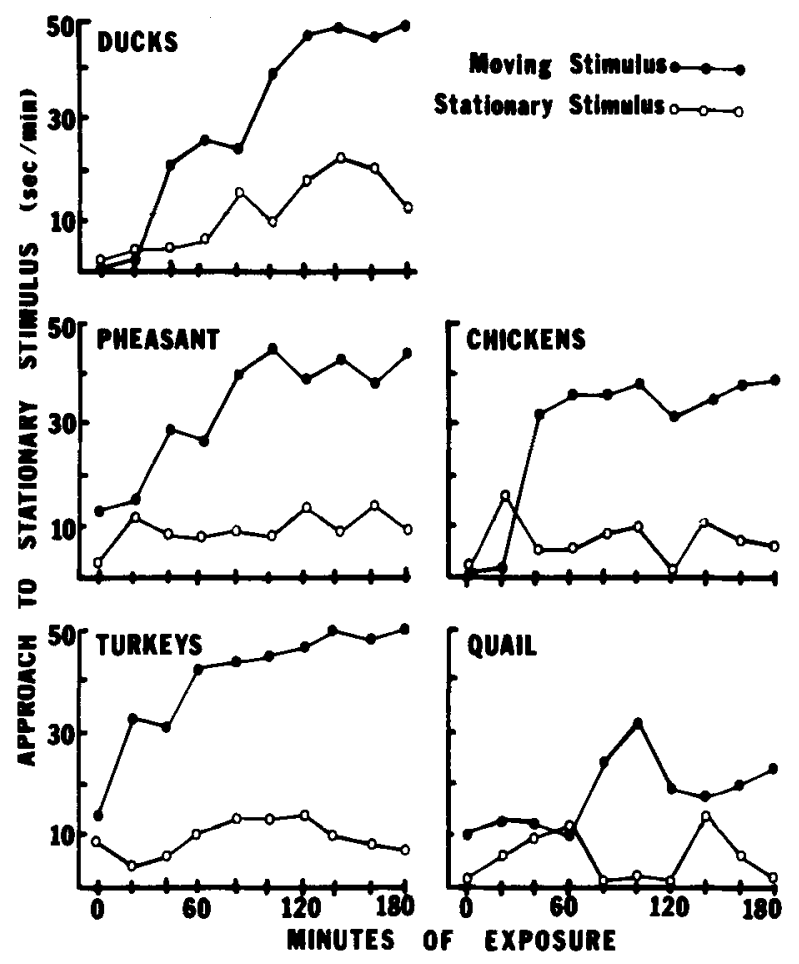

Figure 1. Mean seconds of approach by each species during est presentations of the stationary stimulus, as a function of vether subjects received exposure to the moving stimulus or to nly the stationary stimulus. Exposure duration is cumulated Icross nine 20-min sessions.

species. In the present experiment, either an overall iffect of exposure condition (i.e., moving vs. staionary) or an interaction between the effects of exposure condition and exposure duration would mply that significant acquisition had occurred in sne group of subjects relative to the other.

The duck data yielded a significant interaction $[F(9,54)=27.58, p<.01]$ and a nonsignificant overall effect of exposure condition $[F(1,6)=1.58, p>.10]$. The pheasant data yielded a significant interaction $[F(9,54)=19.17, p<.01]$ and a marginally signifizant effect of exposure condition $[F(1,6)=5.12$, $\mathrm{p}<.07$. The chicken data also yielded a significant interaction $[F(9,90)=4.87, p<.01]$ and a marginally significant effect of exposure condition $[F(1,10)=$ 4.77, $\mathrm{p}<.06$ ]. The turkey data yielded a significant interaction $[F(9,90)=3.50, p<.01]$, as well as a significant effect of exposure condition $[F(1,10)=44.44$, $\mathrm{p}<.01]$. The quail data yielded a nonsignificant interaction $[F(9,72)=1.94, p>.10]$ but a significant effect of exposure condition $[F(1,8)=5.72, p<.05]$. Each of the five species, then, generated either a significant interaction or a significant overall effect of exposure condition.

The overall effect of exposure duration was significant at the .01 level for the ducks $[F(9,54)=31.81]$, the pheasant $[F(9,54)=27.50]$, the chickens $[F(9,90)$
$=4.24]$, and the turkeys $[F(9,90)=8.04]$, but was nonsignificant for the quail $[\mathrm{F}(9,72)=.89, \mathrm{p}>.10]$.

\section{DISCUSSION}

Although it may have been preferable to use the same imprinting stimulus for all five species, such uniformity was not prerequisite to achieving the goal of the present research. Our question was, given a moving object (any moving object) that is able to elicit filial behavior from a given species, and given that the birds will not respond to the object when it is stationary, will the static visual characteristics of the object acquire behavioral control in those birds that see the object in motion? It was not our purpose to directly compare all five species except in the very broadest of terms (i.e., whether or not each species showed the acquisition phenomenon). Indeed, more specific comparisons (e.g., whether ducks approached the stimulus more quickly, or at a higher asymptotic level, than the chickens) would not even be possible without extensive preliminary investigation of potential species differences in preferred stimulus characteristics (sound, color, size, shape, pattern of movement). Put another way, it is unlikely that any single imprinting stimulus would be equally attractive to all five species, and this fact must necessarily hinder attempts at detailed species comparisons.

In all species, the $M$ subjects showed a greater tendency to approach the stationary stimulus than did the S subjects, which, in turn, displayed only weak approach tendencies throughout the experiment. In the case of the quail, neither the difference between the $M$ and $S$ subjects nor the overall approach tendency of the $M$ subjects was as marked as in the other species. No doubt the inability of the moving stimulus to elicit strong approach in the $M$ quail accounts for the weak control by the stationary stimulus in those subjects. Why the quail responded erratically to the moving stimulus, however, is unclear. The high mortality of quail in the present experiment may mean that the surviving subjects were themselves under some physiological stress that interfered with the imprinting process. It is also true that other investigators of Japanese quail have reported difficulty both in using approach responses (as opposed to distress vocalization) as an index of filial attachment and in demonstrating strong imprinting to a visual (as opposed to auditory) stimulus (Evans \& Coseus, 1977; Green \& Adkins, 1975). In any event, despite the weakness of the effect, the finding that $M$ quail approached the stationary stimulus more than the $S$ quail is basically consistent with the results for the other four species.

The acquisition of behavioral control by initially neutral features of an imprinting object appears to be a robust phenomenon that can be demonstrated 
in a variety of different experimental contexts. It has been documented with several different types of imprinting objects (Eiserer, 1980; Hoffman et al., 1972); it applies to the initially neutral auditory as well as static visual features of an imprinting object (Eiserer \& Hoffman, 1974; Hoffman et al., 1972); it can be assessed through a variety of filial behaviors, including suppression of distress calls (Hoffman et al., 1972), approach responses (Eiserer, 1980), operant responses (Eiserer et al., 1975), and-if Klopfer's (1965) findings are accepted as relevant-choice behavior; it can be seen in imprinting that occurs after as well as during the sensitive period (Gaioni et al., 1978); and, as indicated by the present work, it occurs in a wide variety of precocial birds. Given the empirical inconsistencies that often plague the imprinting literature and given the realization that seemingly important behavioral effects sometimes turn out to be highly dependent upon peculiar experimental parameters (Eiserer, 1978a), the contextual independence of the acquisition phenomenon is reassuring to those who would ponder its underlying processes.

\section{REFERENCES}

Bruning, J. L., \& Kintz, B. L. Computational handbook of statistics. Glenview, Ill: Scott, Foresman, 1977.

Eise RER, L. A. Behavioral control by stimulus components of an imprinting object. Animal Learning \& Behavior, 1977, 5, 153-160.

EISERER, L. A. Anomalous effects in psychology: Parallels between the brain stimulation and imprinting literatures. Psychological Record, 1978, 28, 557-570. (a)
Eiserer, L. A. Maltreatment effects and learning processes in infantile attachment. Behavioral and Brain Sciences, 1978, 3, 445-446. (b)

Eiserer, L. A. Development of filial attachment to static visual features of an imprinting object. Animal Learning \& Behavior, $1980,8,159-166$.

Eiserea, L. A., \& Hoffman, H. S. Priming of ducklings' responses by presenting an imprinted stimulus. Journal of Comparative and Physiological Psychology, 1973, 82, 345-359.

Eiserer, L. A., \& Hoffman, H. S. Acquisition of behavioral control by the auditory features of an imprinting object. Animal Learning \& Behavior, 1974, 2, 275-277.

Eiserer, L. A., Hoffman, H. S., \& Klein, S. H. Persistence of acquired behavioral control in the context of imprinting. Journal of the Experimental Analysis of Behavior, 1975, 24, 255-266.

Evans, R. M., \& Coseus, S. Selective control of peep vocalizations by familiar sound in young Coturnix quail. Behaviour, $1977,62,35-49$.

Gaioni, S. J., Hoffman, H. S., DePaulo, P., \& Stratton, V. N. Imprinting in older ducklings: Some tests of a reinforcement model. Animal Learning \& Behavior, 1978, 6, 19-26.

Green, J. A., \& Adkins, E. K. The effects of prenatal and postnatal auditory stimulation on early vocalization and approach behavior in the Japanese quail (Coturnix coturnix japonica). Behaviour, 1975, 52, 145-154.

Hoffman, H. S., Eiserer, L. A., \& Singer, D. Acquisition of behavioral control by a stationary imprinting stimulus. Psychonomic Science, 1972, 26, 146-148.

Hoffman, H. S., Stratton, J. W., Newby, V., \& Barrett, J. E. Development of behavioral control by an imprinting stimulus. Journal of Comparative and Physiological Psychology, 1970, 71, 229-236.

Klopfer, P. H. Imprinting: A reassessment. Science, 1965, 147, 302-303,

(Received for publication July 12, 1979; revision accepted March 3, 1980.) 